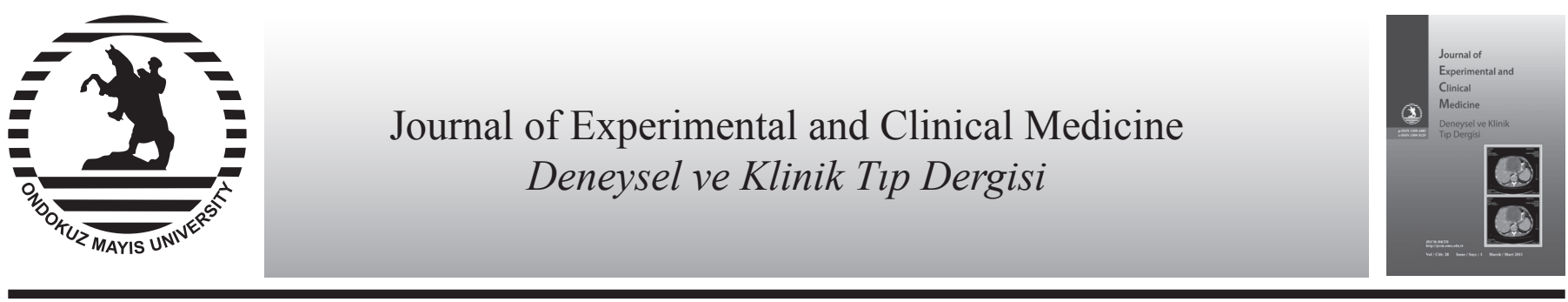

Case Report

\title{
Neck localized cutis laxa
}

\author{
Umut Tuncel*a, Aydın Turan $^{\mathrm{a}}$, Fatma Markoçc, Naci Kostakoğlua \\ ${ }^{a}$ Department of Plastic Reconstructive and Aesthetic Surgery, Medical Faculty, Gaziosmanpasa University, Tokat, Turkey \\ ${ }^{b}$ Department of Pathology, Medical Faculty, Gaziosmanpasa University, Tokat, Turkey
}

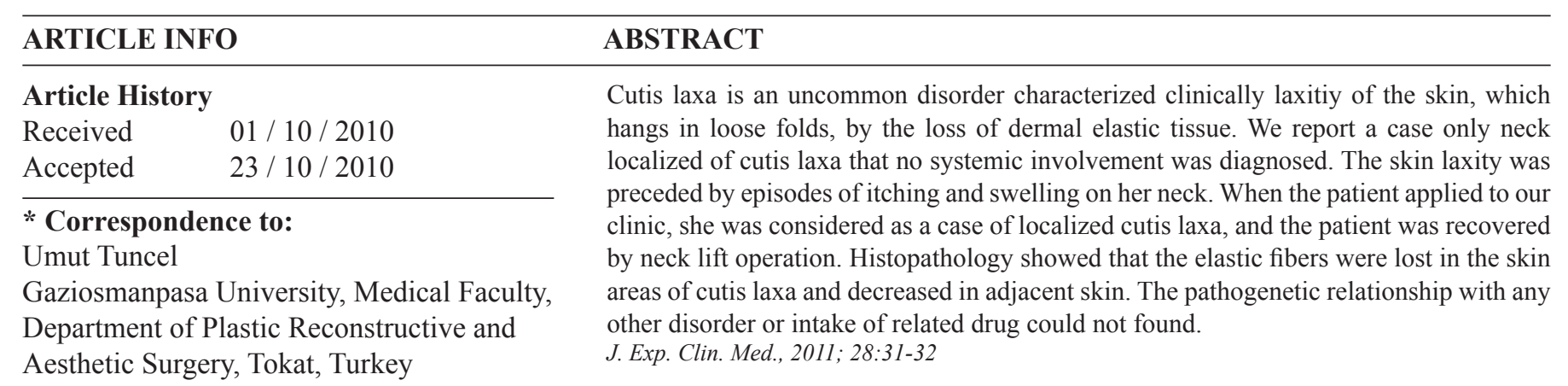

e-mail: drumuttuncel@gmail.com

\author{
Keywords: \\ Localized cutis laxa \\ Elastolysis \\ Neck \\ Skin laxity \\ Elastic fibers \\ Pendulous skin
}

(C) 2011 OMU All rights reserved

\section{Introduction}

A rarely reported cutaneous change is acquired cutis laxa, either as a generalized or localized process. Cutis laxa, also called elastolysis, presents with soft redundant folds of skin that are inelastic, and histology shows sparse, short and fragmented elastic fibres. We present a 23-year-old woman with neck localized cutis laxa. In our case skin biopsies were taken and histopathologic examination revealed that dermal elastic fibers were markedly decreased. These pathologic findings were compatible with cutis laxa. The clinical findings suggested to the localized type of the desease.

\section{Case report}

A 23-year-old female patient was admitted to our hospital, due to complaints of itching and swelling on her neck and nape (Fig. 1a, b). The skin was dark pink and hung loosely. The complaint has continued for two years. Aside from the neck skin pathology, no particular abnormality was noted on the physical evaluation, and laboratory tests including complete blood count, total protein, $\beta 2$-microglobulin, IgG, IgM, C3, C1q and antinuclear antibody (ANA). The other physical examination was also normal. Neck lift surgery was performed and the patient has respectively been recovered by surgery at 4 week. Skin biopsies were taken and histopathologic examination revealed that dermal elastic fibers were markedly decreased (Fig. 2a, b). These pathologic findings were com- patible with cutis laxa. The clinical findings suggested to the localized type of the desease.
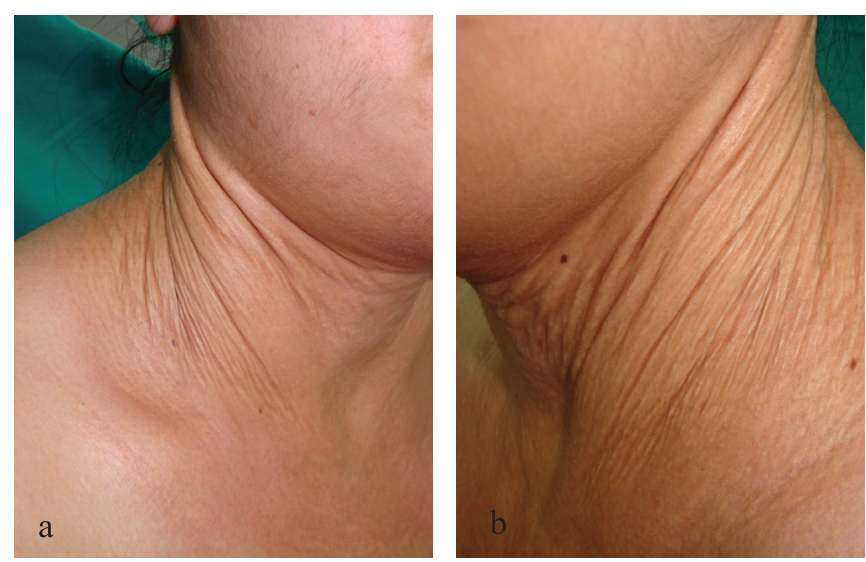

Fig. 1a,b. Preoperative right and left lateral appearance of the patient showing that pendulous skin with a wrinkled surface on her neck and nape.

\section{Discussion}

Cutis laxa that is dermatochalasis or generalized elastolysis is a rare condition characterized clinically by loose pendulous skin and histologically by the loss of dermal elastic fibers and, the disorder may be inherited or acquired, both forms being generalized or localized (Rongioletti et al., 2002; Lucas 
et al., 2009). To our knowledge, the only neck localization of the desease is extremely uncommon in the literature.

The acquired form of the disorder is very rare, that is may occur about 30 cases have been presented (Rongioletti et al., 2002). Some cases have been preceded by hypersensitivity reactions to penicillin and the others have been associated with myeloma, cutaneous lymphoma, systemic lupus erythematosus, nephrotic syndrome and celiac disease (Rongioletti et al., 2002; Appiah et al., 2008; Lucas et al., 2009). Cutis laxa refers to a heterogeneous group of diseases with a characteristic clinical appearance of premature aging. These conditions are distinguished by the age of onset of dermatologic signs whether congenital or acquired, extension of cutaneous involvement, presence of systemic compromise, and pattern of inheritance (Riveros et al., 2004). There have been a few reports of acquired form of disease associated with multiple myeloma and furthermore there are only 2 reported cases of myeloma associated with the acral localized type (Appiah et al., 2008). In this type of the disorder the amyloid deposition was seen surrounding dermal vessels and elastic tissue revealed diminution of elastic fibers around dermal vessels.
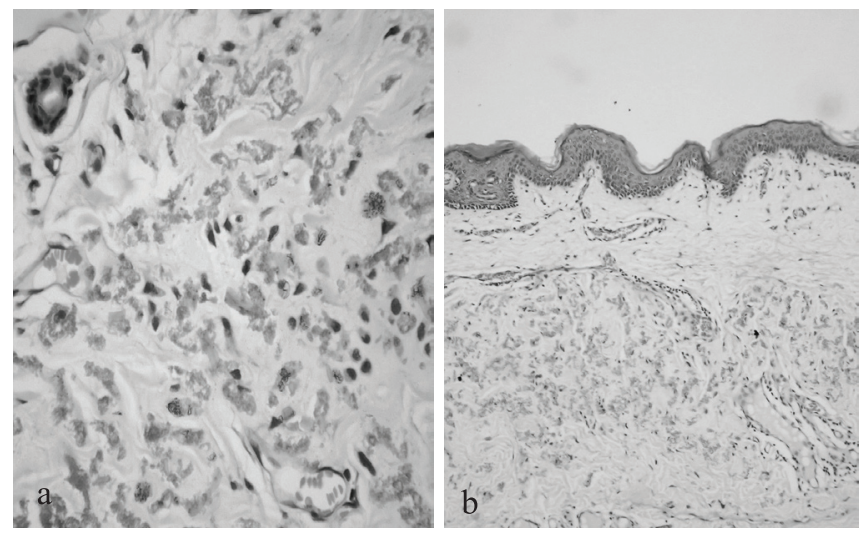

Fig. 2 a,b: Neck skin biopsy shows loose, swollen and itching skin. Biopsy of the central of the lesion shows decrease and deformation of elastic fibers from the dermis and subcutaneous tissue septae (original magnification $\mathrm{x} 40$ ).

The congenital form may be inherited as an autosomal recessive disorder, with severe pulmonary and cardiovascular involvement and early death. Autosomal dominant form is a relatively benign with largely skin manifestations and few complications; and as a rare X-linked recessive variant (Banks et al., 2003; Ozsoy et al., 2007; Morava et al., 2009). Patients with either the recessive or dominant forms have characteristic faces with a hooked nose, everted nostrils and a long upper lip (Riveros et al., 2004; Appiah et al., 2008).

The diagnosis is suggested clinically, but requires histopathologic confirmation (Morava et al., 2009). The most common finding is a marked reduction of elastic fibers at all levels of the skin, with the residual fibers being fragmented and of irregular thickness (Banks et al., 2003; Morava et al., 2009). However, collagen fibers are usually normal. In the postinflammatory variant, an infiltrate with neutrophils, lymphocytes and histiocytes can be demonstrated close to the affected elastic fibers (Banks et al., 2003). Most routine laboratory tests such as serum elastase, serum elastase inhibitor, serum copper and ceruloplasmin, alpha1-antitrypsin and lysyl oxidase levels are usually normal and might be helpful, but they are not required for the strongly diagnosis.

Differential diagnosis should be made between acquired cutis laxa and other cutis laxa syndromes, such as postinflammatory elastolysis and cutis laxa, anetoderma, EhlersDanlos syndrome, Marfan syndrome, and pseudoxanthoma elasticum (Banks et al., 2003; Nahai, 2003). There is no satisfactory treatment for cutis laxa. Medical treatment such as dapsone was given to patients with the inflammatory phase and was able to control swelling. However, it could not prevent progression of the disease (Banks et al., 2003). The role of plastic surgical repair seems to be the only hope for these patients and has been recognized since the late 1940s (Banks et al., 2003). Rhytidectomy or neck lift surgery is often the first surgery proposed and other procedures such as earlobe reduction, blepharoplasty, correction of lower eyelid laxity and upper eyelid ptosis could be beneficial and as is known the skin wound healing is normal in cutis laxa (Nahai, 2003; Ozsoy et al., 2007). However, according to our knowledge from the literature, in many cases no improvement is seen and unsuccessful surgeries are not rare. These patients should be informed that repeating surgeries in short intervals may be necessary and that better results are usually obtained after repeat operations (Nahas et al., 1999; Riveros et al., 2004).

Our patient is a highly rare case of localized cutis laxa who have skin lesions on her neck and nape localization. Skin biopsies were taken and histopathologic examination revealed that dermal elastic fibers were markedly decreased. These pathologic findings referred to cutis laxa and the clinical findings suggested to the localized type of the desease. In conclusion, it should know that cutis laxa is an unusual condition and should be helpmate to patients with cutis laxa about phsychological and social support.

\section{REFERENCES}

Appiah, Y.E., Onumah, N., Wu, H., Elenitsas, R., James, W. 2008. Multiple myeloma-associated amyloidosis and acral localized acquired cutis laxa. J. Am. Acad. Dermatol. 58, 32-33.

Banks, N.D., Redett, R.J., Mofid, M.Z., Manson, P.N. 2003.Cutis laxa: clinical experience and outcomes. Plast. Reconstr. Surg. 111, 24342442.

Lucas, A., Banuls, J., Mataix, J., Crespo-Perez, M. 2009. Localized acquired cutis laxa secondary to interstitial granulomatous dermatitis. Clin. Exp. Dermatol.34, 102-105.

Morava, E., Guillard, M., Lefeber, D.J., Wevers, R.A. 2009. Autosomal recessive cutis laxa syndrome revisited. Eur. J. Hum. Genet. 17, 10991110 .

Nahai, F. 2003. Cutis laxa: clinical experience and outcomes. Plast. Reconstr. Surg. 111, 2443-2444.

Nahas, F.X., Sterman, S., Gemperli, R., Ferreira, M.C. 1999. The role of plastic surgery in congenital cutis laxa: 10-year follow-up. Plast. Reconstr. Surg. 104, 1174-1178.

Ozsoy, Z., Gozu, A., Dayicioglu, D., Mete, O., Buyukbabani, N. 2007. Localized cutis laxa and blepharoplasty. Dermatol. Surg. 33, 1510-1512 Rongioletti, F., Cutolo, M., Bondavalli, P., Rebora, A. 2002. Acral localized acquired cutis laxa associated with rheumatoid arthritis. J. Am. Acad. Dermatol. 46, 128-130.

Riveros, C.J., Gavilán, M.F., França, L.F., Sotto, M.N., Takahashi, M.D. 2004. Acquired localized cutis laxa confined to the face: case report and review of the literature. Int. J. Dermatol. 43, 931-935. 